\title{
El trabajo con familias de menores infractores: elemento clave en el proceso inclusivo
}

\author{
Nuria López Roca $\left(^{*}\right)$, María Fernández Hawrylak(**), Jesús Soldevila Pérez( $\left.{ }^{* * *}\right)$, Joan Jordi Muntaner Guasp ${ }^{* * * *}$ ) \\ ${ }^{(*) I E S ~ C a n ~ B a l o,}\left({ }^{* *}\right)$ Universidad de Burgos, $\left.{ }^{* * *}\right)$ Universidad de Vic-Universidad Central de Cataluña, $\left.{ }^{* * *}\right)$ Universidad de las Islas Baleares.
}

\begin{abstract}
RESUMEN
La familia y las relaciones familiares en la vida de quienes han cometido delitos es un tema clave en los procesos de intervención educativa y social. El artículo que se presenta argumenta la importancia de la implicación de la familia en el trabajo de reinserción al contexto familiar, escolar y social habituales, desde una perspectiva comunitaria, de los menores/jóvenes infractores que tienen que cumplir medidas judiciales de internamiento firme o cautelar; y describir el Programa de Intervención Familiar (PIF) que desarrolla el equipo técnico-educativo de un Centro Socioeducativo de Palma de Mallorca (España). En él se hace un trabajo de reorganización de las relaciones familiares y sociales para lograr nuevas pautas de funcionamiento más funcionales y constructivas, labor reforzada mediante otros programas transversales. Se trata de un trabajo descriptivo donde se justifican los beneficios tras la intervención familiar desde el Programa, diseñado y desarrollado según el modelo sistémico. Las familias participantes lo valoran positivamente, atribuyen mejoras en las relaciones familiares, y resaltan la evolución de los menores en torno al reconocimiento del su rol como alumnado competente. Estos jóvenes no pueden ser tratados de forma aislada si se quieren tener garantías de éxito en su reinserción, sino dentro de un marco comunitario donde la familia desempeña un papel clave en dicho proceso.
\end{abstract}

Palabras Clave: familia, menores infractores, intervención familiar, educación inclusiva.

\section{Working with families of juvenile offenders: keystone in the inclusive process.}

\section{ABSTRACT}

Family and family relationships of those who had committed a crime is a key issue in the processes of educational and social intervention. We justify the importance of the family involvement in the reintegration of juvenile offenders who must serve judicial measures of final or precautionary internment to their regular family, scholar and social context from a community perspective. We describe the Program of Family Intervention developed by the technical and educational team of a socio-educational center of Palma de Mallorca (Spain). A work of reorganization of family and social relationships is made in this center in order to achieve new and more functional and constructive operating patterns among the family members. Other cross-disciplinary programs reinforce this work. This paper is a descriptive work in which we justify the benefits of the intervention from the Program, that has been designed and developed according to the Family Systemic Model. The families that take part in the Program value it positively, attribute improvements in family relationships to it, and highlight the evolution of minors on their own recognition of their role as skilled students. These teenagers cannot be treated in isolation if we want to ensure their reintegration success, but within a community framework in which the family develop a key role in the process.

Keywords: family, juvenile offenders, family intervention, inclusive education.

\section{Introducción}

La importancia de la familia y de las relaciones familiares en la vida tanto de las/los jóvenes que han cometido delitos como de los delincuentes adultos es un tema clave, especialmente en los procesos de intervención educativa y social.

Ninguna familia es igual a otra. En cada una rigen normas y reglas particulares que permiten organizar las funciones que llevarán a cabo sus miembros; ese interactuar por medio de funcio- nes garantiza que el sistema se mantenga estable, prescribiendo y estableciendo los límites para la conducta de sus miembros. La familia, en su dinámica, vive unos procesos interaccionales comunes, pero con una configuración particular (Amarís, Amar, y Jiménez, 2005).

La familia es un agente primario de socialización, donde los menores se desarrollan intelectual y emocionalmente, y donde adquieren los valores sociales. Pero también puede ser generadora de dificultades $\mathrm{u}$ obstaculizar el proceso de crecimiento, 
maduración y socialización de sus miembros más jóvenes. Hay pruebas por ejemplo de que un clima familiar negativo y un bajo apoyo familiar pueden conducir a depresión tanto a niños como a adolescentes (Ibabe, Arnoso, y Elgorriaga, 2014).

La premisa que subyace a una aproximación socioecológica es que el comportamiento antisocial grave y persistente es multicausal. Por tanto, las intervenciones efectivas se dirigen a múltiples fuentes de comportamiento delictivo. Tales fuentes se encuentran no sólo en el joven (valores y actitudes, habilidades sociales y factores biológicos), sino también en el contexto donde se desenvuelve (la familia, la escuela, el grupo de amigos y el vecindario). Los factores de riesgo asociados con el comportamiento antisocial grave y persistente se han identificado a lo largo de décadas de investigación longitudinal (Fonagy et al., 2013), e incluyen: impulsividad y sobreactividad en el lado del joven; bajos niveles de implicación parental y un estilo educativo crítico y riguroso; altos niveles de conflicto familiar y ruptura; y asociaciones del joven con otros compañeros de conducta problemática.

Algunos autores_(Ibabe et al., 2014; Ibabe y Jaureguizar, 2011; Sanz et al., 2004) han descrito también cómo el uso continuado de drogas puede llevar a situaciones de conflicto familiar como consecuencia de dicho uso (bajo rendimiento académico, problemas de dinero, o salir por la noche hasta tarde), y una mayor agresividad en las relaciones interpersonales y familiares.

Al hablar del menor con problemas psicosociales se está haciendo mención a jóvenes que, por emerger de un contexto sociofamiliar adverso caracterizado por la disfuncionalidad, han desarrollado patrones adaptativos para la sobrevivencia, aunque ni funcionales ni sanos para su bienestar psicológico. Dichos patrones les permiten sobrevivir en estos contextos, bajo la percepción constante de amenazas, desconfianza y falta de apoyo por parte de las instituciones y personas del entorno. En ambientes opresivos y malignos desarrollarán pautas alternativas de adaptación, más apropiadas a las situaciones de su vida. El sistema familiar representa para el menor ese ambiente social que puede constituirse en reforzante o amenazador y agresivo, según sea la vivencia de sus protagonistas. Como resultado de contextos familiares disfuncionales y amenazantes se encuentran jóvenes con problemas psicosociales que muestran sus dificultades en torno a las competencias psicosociales para afrontar la vida (Amarís et al., 2005).

Muchos estudios argumentan la importancia de involucrar a las familias y a otros agentes comunitarios para garantizar el éxito educativo (Egido, 2015; Garreta, 2017; López, 2014) y la mejora de las relaciones familiares (Cagigal, 2009). En la práctica con adolescentes infractores también son varios los estudios que destacan como vital el tener un objetivo comunitario más que individual, resaltando en particular una intervención que respete la importancia de los lazos familiares y comunitarios (Funes, 1992; Trotter, Baidawi, y Evans, 2015; Vázquez, 2012).

Es por ello fundamental el trabajo conjunto entre un experto en intervención familiar y el cuidador del joven infractor para mejorar las habilidades parentales de los progenitores, aumentar las relaciones familiares, incrementar el apoyo de las redes sociales, fomentar la asistencia a clase y los logros del adolescente, y reducir la asociación del joven con compañeros delincuentes. Este trabajo con familias debe ir dirigido a (Trotter et al., 2015): identificar alguna conexión o lazo común con una persona significativa de la familia extensa (o contexto) para construir el entendimiento y la confianza entre los jóvenes y sus familias; elaborar una estrategia de aproximación al joven basada en un enfoque familiar; y establecer unos conceptos claros sobre la naturaleza y el propósito de la intervención (y si es posible una alianza positiva) que incluya a la familia o al tutor.
Desde hace unos años, las directrices internacionales definidas y promovidas por organismos y entidades como la UNES$\mathrm{CO}$, la OCDE o la ONU, son claramente favorables a la educación inclusiva. El ímpetu que estas instituciones han depositado en la educación inclusiva parte del hecho de que se entiende la educación como instrumento de inclusión social, como promotora de una ciudadanía respetuosa con los derechos de todas las personas, y que por lo tanto convive en igualdad de oportunidades. Ésta ha sido también sin duda la voluntad y el anhelo de muchas de las familias. Con esta finalidad las investigaciones en educación tienen que estar al servicio de las personas, y contribuir a transformar de forma innovadora las situaciones que viven los niños y los jóvenes infractores y sus familias.

La igualdad de oportunidades es un principio que a menudo ha sido mal interpretado. Esta interpretación seguramente proviene de la versión que se pone en práctica del concepto. Lema (2010) señala que, con una versión minimalista de este principio, con la simple universalización de los sistemas educativos, ya se consideraría que se está promoviendo este derecho. Ahora bien, con una versión más amplia no sería suficiente con este requisito mínimo, y por lo tanto estaríamos lejos todavía de garantizarlo en las condiciones actuales. Es importante no perder de vista que, para preservar la igualdad de oportunidades, lo que en realidad hace falta que se ponga en práctica es la equidad. En este sentido, las directrices de los centros tienen que ir dirigidas a garantizar las medidas necesarias para que se pueda desarrollar una educación inclusiva y de calidad para todos y cada uno de sus integrantes, independientemente de sus condiciones de partida. Una educación, por tanto, que pone los esfuerzos en contribuir a una sociedad más justa (Ainscow, Dyson, Goldrick, y West, 2012). Así pues, el niño y el joven infractor tendrían que poder encontrar las condiciones necesarias para su desarrollo y bienestar en los entornos ordinarios, de forma que su inclusión fuera efectiva y pudieran disfrutar de una vida lo más normalizada posible y en igualdad de oportunidades.

En este artículo se expone específicamente el trabajo que en un Centro Socioeducativo de las Islas Baleares, España, se desarrolla con las familias para que menores infractores en situación de cumplimiento de medidas judiciales vuelvan a ser alumnos exitosos. Desde el Programa de Intervención Familiar (PIF) se hace un trabajo continuo y cíclico de reorganización de las relaciones sociales y familiares, cuyo objetivo es conseguir nuevas pautas de funcionamiento más funcionales y constructivas entre los miembros de la familia. Desde otros programas se refuerza esta labor de forma transversal, con el fin de apoyar la intervención que se lleva a cabo con las familias. Todo ello tiene una sólida base teórica, reflejo de los años de experiencia y del sentir común del grupo de trabajo que lleva adelante este proyecto (López, 2006; López y Muñoz, 2007). Los protagonistas de esta experiencia son jóvenes (también alumnas/os) con unas necesidades educativas y personales muy específicas, que vivirán fuera de su contexto familiar, escolar y social durante un tiempo (semanas, meses o años, según cada caso). Esto no significa que se les desvincule de ese círculo: el objetivo es trabajar con todos sus referentes contextuales para que el retorno se realice con la mayor garantía de éxito, lo que hace imprescindible un trabajo coordinado con todos los agentes implicados.

\section{Marco normativo y líneas de intervención psicosocial con los menores infractores y sus familias}

Muchas veces la problemática psicosocial que afrontan estas/ os jóvenes, menores infractores, es el resultado de sus circunstancias sociales y familiares. Sin embargo, para obtener un pa- 
norama claro sobre estas condiciones es necesario definir y diferenciar la problemática que presentan, considerándola incluso desde la perspectiva legal.

El éxito y el fracaso suelen ir ligados a la pertenencia a un grupo social y al nivel cultural y/o económico. Las élites siempre han gozado de mayor éxito académico y más oportunidades sociales. No obstante, la tendencia en los últimos años ha variado por la incompetencia de algunas familias para dar respuesta a unas/os hijas/os poco habituados a las exigencias del entorno. El fracaso académico suele acentuarse a partir de la educación secundaria, donde las instituciones pensadas para dar respuesta a las desigualdades se van convirtiendo cada vez más en excluyentes, y donde, al margen de su finalidad, van separando al alumnado exitoso del que no consigue superar las exigencias que el sistema ha generado. Esta situación no es fácilmente modificable. La educación inclusiva, por contra, supone adoptar otro tipo de pensamiento y una postura crítica justamente contraria a la teoría y la práctica educativas basadas en la selección, la competición y el individualismo (Barton, 2009). La educación inclusiva huye del instrumentalismo, que aprovecha la educación para conseguir los objetivos económicos de la sociedad, favoreciendo la enseñanza y el aprendizaje de forma competitiva y jerárquica, seleccionando y diferenciando los alumnos de modo que conduce a la reproducción de las desigualdades (Barton, 2003). En la educación inclusiva "la consecución de un objetivo curricular concreto no siempre es la única, ni tan siquiera la principal, meta para garantizar el éxito y la felicidad en la vida" (Stainback, Stainback, y Moravec, 1999, p. 85).

La Constitución Española (1978) reconoce en su artículo 27 el derecho a la participación efectiva de todos los sectores afectados en la educación, mientras que la Ley Orgánica 8/2013, de 9 de diciembre, para la mejora de la calidad educativa (LOMCE), pone el foco de atención en la familia cuando subraya que "las familias son las primeras responsables de la educación de sus hijos y por ello el sistema educativo tiene que contar con la familia y contar con sus decisiones". Por su parte, el Decreto 34/2015, de 15 de mayo, por el que se establece el currículo de la Educación Secundaria Obligatoria en las Islas Baleares, de conformidad con la Ley Orgánica 8/1985, de 3 de julio, reguladora del Derecho a la Educación, reglamenta en su artículo 32 la participación de los padres y tutores legales en el proceso educativo. Reconoce también en sus Anexos, dedicados a la estructuración del currículum a lo largo de las diferentes materias, la importancia del acompañamiento a la familia, así como su intervención en el desarrollo del currículum y el apoyo a sus hijos en la evolución del proceso educativo, y determina que sean los centros educativos los que proporcionen las condiciones necesarias para su cumplimiento. Es importante remarcar que en su artículo 13 establece la necesidad de fomentar "el aprendizaje de la prevención y la resolución pacífica de conflictos en todos los ámbitos de la vida personal, familiar y social [...]". En esta misma dirección, en el artículo 8.8 sobre el proceso de aprendizaje y atención personalizada, prescribe que el centro va a contribuir a este proceso desde la acción tutorial, implicando a los alumnos y sus familias en el funcionamiento del centro.

En materia penal, la Ley Orgánica 5/2000, de 12 de enero, reguladora de la responsabilidad penal (LORPM), es la ley vigente que se aplica a menores infractores en España. Considera menores infractores a aquellas personas jóvenes, entre los 14 y los 18 años de edad, que cometen un hecho tipificado como delito o falta en el Código Penal o las leyes penales especiales. Las medidas que se adoptan están orientadas hacia la efectiva reinserción social, estableciendo la competencia de las Comunidades Autónomas para su ejecución, bajo el control del Juez de Menores.
Asimismo, en su artículo 7 define las medidas susceptibles de ser impuestas a los menores y las reglas generales de determinación de las mismas.

Durante el cumplimiento de estas medidas existe el compromiso por parte de los centros socioeducativos, donde se ejecutarán éstas, de desarrollar los programas necesarios para garantizar procesos de retorno al contexto social de manera exitosa y positiva. Uno de esos programas es el que describe este artículo dirigido al trabajo con familias desde una perspectiva integral.

La familia, la escuela y la comunidad deben elaborar programas integrales de atención a los menores para prevenir las situaciones de riesgo, que proporcionen herramientas que les ayuden en su competencia social para mejorar en todos los ámbitos de su vida (Germán y Ocáriz, 2009; Vásquez, 2003), realizando dinámicas conjuntas dirigidas a optimizar la comunicación y la relación entre hijos y familias (Rey, 2014).

Pero en nuestro contexto son pocos los estudios relativos a la escuela y menores infractores o centros de justicia juvenil que incluyen a la familia como parte de los mismos. La línea general son programas dirigidos al afrontamiento de problemas específicos (consumo de tóxicos, reducción de conducta antisocial, o violencia filio parental entre otros), y basados en una intervención muy fraccionada donde se suele trabajar con familias y menores, pero usualmente no de manera unificada. Tampoco se han encontrado programas en los que se incluya como una parte importante al profesorado del ámbito de enseñanza reglada. No debemos olvidar que los menores infractores se encuentran en edad escolar (obligatoria o post-obligatoria), por lo cual hablar de una intervención global es incluir el ámbito escolar. Es importante recordar que el objetivo final es la reinserción exitosa del menor en su contexto escolar, familiar y social.

El diseño de programas que implican a la familia para tratar de disminuir las dificultades que pueden darse dentro de la dinámica familiar, y que llevan al internamiento de alguno de sus miembros menores o jóvenes, y a generar pautas más funcionales de relación, pasaría por conocer cuáles son las características que definen la estructura y funcionamiento en este tipo de familias. De forma general podrían señalarse:

- Reglas o normas: ausencia de reglas y normas, y cuando las hay son normas implícitas y metarreglas.

- Comunicación: falta de comunicación por escasa presencia de las figuras paternas en el hogar (si no están, no se comunican), hostilidad y agresividad verbal (discusiones, gritos, amenazas, insultos e indiferencia).

- Dinamismo: límites difusos entre subsistemas parental y filial; incapacidad para reconocer, atribuir y manifestar la importancia de cada miembro para el otro; incapacidad para negociar; falta de apoyo, unión y convivencia; pérdida considerable de valores familiares (actividades en familia de tipo doméstico, reuniones familiares escasas y cuando las hay son hostiles...); desintegración familiar.

- Actitudes educadoras: la representación de la autoridad suele estar centrada en la madre y en otros miembros de la familia como los tíos. Los estilos educativos son autoritarios y carentes de afectividad, lo que contribuye a generar un ambiente familiar caracterizado por la agresividad, y a construir procesos sociales inestables en los cuales la imagen del delito es resignificada por los actores sociales como flexible frente a las exigencias del día a día caracterizado por la violencia o el maltrato (Amarís et al., 2005; Fonagy et al., 2013). También pueden existir pautas educativas indiferentes, propias de padres con escasa implicación en la crianza y la educación (Fonagy et al., 
2013), fríos y distantes con sus hijos, poco sensibles a sus necesidades; y ausencia de normas, pero en ocasiones el control que ejercen es excesivo sometiéndoles a fuertes castigos sin mediar ninguna explicación o razonamiento de la conducta indebida, por lo que el menor no acata normas, y se convierte en insensible a las emociones de los demás (baja empatía) y muy vulnerable a conflictos personales y sociales.

Igualmente, cada contexto escolar y social tiene sus propias características que deben conocerse y concretarse. Ello induce a pensar que construir la capacidad para desarrollar una implicación de las familias en los centros que se encargan de atender a jóvenes precisa de la creación de una comunidad cívica entre familias y centros, pues estos últimos funcionan si tienen como contexto ecológico la comunidad (Bolívar, 2006). Por ello es necesario diseñar proyectos y prácticas innovadoras que permitan a la escuela trabajar con las familias y la comunidad de manera conjunta, generando relaciones de calidad en la consecución del éxito y la felicidad de los individuos. Una de las bases del programa que presentamos es un trabajo paralelo entre los implicados en una primera fase, pero con actuaciones conjuntas que conectan a profesionales, menores y familias en fases posteriores.

\section{Programa de Intervención Familiar (PIF) dirigido a familias de menores infractores}

El trabajo que se presenta se contextualiza en uno de los tres Centros Socioeducativos donde se ubican aulas de un Instituto de Enseñanza Secundaria de Palma de Mallorca, vinculado a través de un convenio a la Fundación S'Estel del Gobierno Balear que gestiona los centros de menores en las Islas Baleares. Este Centro ejecuta medidas privativas de libertad marcadas por la LORPM. Acoge a estudiantes de entre 14 y 21 años (ya no son menores, pero han delinquido antes de cumplir la mayoría de edad) que tienen que cumplir una medida judicial de internamiento firme o cautelar en régimen: a) cerrado; b) semiabierto; c) abierto; y d) internamiento terapéutico en régimen cerrado, semiabierto o abierto. Actualmente tiene una capacidad para 17 plazas.

No es un centro escolar que abre unas horas al día o que se dedica sólo a la formación académica, sino de un centro de internamiento activo las 24 horas del día donde las/los jóvenes van a vivir a tiempo completo durante un período mínimo de tres meses, con lo cual estarán fuera de su contexto familiar, escolar y social habituales.

El Centro tiene como finalidad la ejecución de todas las medidas de internamiento privativas de libertad. Para ello asume como objetivo principal posibilitar, durante el tiempo que dure la medida, el trabajo con los jóvenes de manera integral para potenciar una inserción social, educativa y/o laboral real y efectiva. No sólo promueve una intervención individual dirigida a fomentar la autonomía, la responsabilidad y el desarrollo moral como los tres pilares básicos, sino que también dirige sus actuaciones hacia una perspectiva contextual donde la familia es uno de los pilares fundamentales a considerar. Con este propósito, durante el curso 2005-2006 implantó el Programa destinado a familias que se describe.

\section{Perfil del alumnado y sus familias}

La adolescencia es un momento de transición delicado y de grandes cambios producidos de una manera acelerada en un periodo cronológico muy corto, puesto que se trata de una etapa de cinco o seis años en el ciclo vital. Implica cambios en diferentes esferas de la vida de las/los jóvenes (biológicos, sociales, personales, etc.). Además, representa una fase trascendental, el tránsito a la vida adulta, con todo lo que ésta supone. La falta de concordancia entre lo que la/el joven necesita y la respuesta que encuentra puede provocar sentimientos de angustia, temor, incertidumbre, etc., que no en pocas ocasiones desembocan en problemas como se ha descrito anteriormente. Si éstos no se canalizan de manera adecuada surgirán los primeros momentos de desadaptación. Consecuencia de ello podemos encontrar el perfil típico de los menores internos en el Centro: adolescentes problemáticos/adolescentes con muchos problemas; menores con problemas graves del comportamiento (Orregoa, Painob, y Fonseca-Pedreroc, 2016); objetores escolares; desarraigados del núcleo familiar; transgresores de normas; inconformistas y "enfadados con el mundo"; en privación de libertad por medida judicial.

En definitiva, se trata de jóvenes de gran potencial, pero con pocas habilidades para poder desarrollarlo. Estos adolescentes, que por diferentes circunstancias han dejado de sentirse como tal, entran en una espiral destructiva para sí mismos. Necesitan un entorno que les valore y crea en ellos para empezar a evolucionar como personas, y también como alumnos. Está claramente demostrado que en el perfil social de estos alumnos destacan la influencia social y los aspectos socioculturales (Marchesi, 2004).

El perfil de las familias de estos jóvenes ha variado de manera considerable en los últimos años, desde familias de estructura pobre e inestable, de minorías étnicas o en condiciones de deprivación ambiental importantes, a familias aparentemente estructuradas, estables, de nivel socioeconómico medio-alto y con un ambiente en principio favorable. Pero, a pesar de este cambio significativo, la mayoría de familias tiene un denominador común que se refleja en los siguientes aspectos: ausencia de límites; pautas educativas inconsistentes (por abandono o por sobreprotección); desarraigo, falta de sentimiento de pertenencia; ausencia de canales de comunicación constructivos; objetivos y/o metas de vida contrapuestos.

En la actualidad, como la población es multicultural, se crea un espacio de intercambio y comunicación entre familias de distintos orígenes, y las actividades conjuntas realizadas posibilitan compartir diferentes valores, visiones educativas...

\section{Perfiles profesionales}

El equipo del Centro está compuesto por un grupo de profesionales cohesionando formado por personal técnico y educativo (educadores y trabajadores sociales, pedagogos, psicólogos, maestros y profesores de secundaria), personal de cocina y limpieza, y el equipo de seguridad. Todos ellos comparten un objetivo común: promover procesos de éxito hacia la reinserción social. Para ello cada uno asume su rol y genera herramientas para ir dando respuesta a las necesidades que se van planteando desde su formación y especialidad. Es la figura del trabajador social la que se implica de manera más directa en el trabajo con las familias, siempre en colaboración con el grupo de profesionales indicados.

\section{Objetivos}

Tal y como se ha ido avanzando, el objetivo principal y meta común de intervención en el Centro es promover, desde un abordaje individual, la educación integral de los jóvenes (que además son alumnos), proporcionándoles las herramientas necesarias que les ayuden a desarrollar sus competencias social, emocional y de vida cotidiana, necesarias para ser capaces de afrontar la 
realidad con los recursos personales apropiados, posibilitando una adecuada y exitosa inserción familiar, escolar y social.

De forma particular, el objetivo familiar que se pretende conseguir en el PIF se ha formulado desde una doble vertiente: individual hacia el menor, y contextual hacia la reorganización del sistema familiar.

\section{Actuaciones (planteadas desde una doble línea de acción)}

\section{A. Dirigidas hacia el propio individuo}

Esta línea de actuación va dirigida a dotar al joven de herramientas y estrategias para su reinserción en su núcleo familiar de referencia, y consecuentemente su reinserción social y escolar a través del desarrollo al máximo de sus capacidades.

B. Dirigidas a la coordinación e intervención con y en el núcleo familiar

Desde esta línea se procura identificar el vínculo más importante para la/el joven, generar y mantener el contacto regular con esa persona, y a ser posible con los demás miembros de la familia, y con el centro escolar de referencia del alumno. El trabajo con la familia va dirigido a reorganizar y reajustar el contexto familiar para que el regreso del menor tenga ciertas garantías de éxito.

\section{Metodología}

Los menores que ingresan en un centro de internamiento por haber cometido actos delictivos sufren un acontecimiento traumático, que supondrá un gran coste emocional tanto para ellos mismos como para su familia. Conseguir la no estigmatización del menor, sino considerarlo como un adolescente con problemas graves de conducta, pero con capacidad para cambiar, y no individualizar en él el problema, concibiéndolo como parte de la dinámica familiar, se torna en una pieza clave en la intervención (Coy y Torrente, 1997).

Es fundamental tener presente que no se trata de familias que solicitan voluntariamente ayuda por entender que el comportamiento de sus hijos desestabiliza el equilibrio familiar, sino que son los profesionales de los centros quienes proponen la intervención individual solamente o incluyen a la familia en la intervención, convirtiéndola en usuaria forzosa por derivación del Juzgado de Menores.

Asimismo, motivar e implicar a la familia dentro del proceso es por tanto el primer paso dentro de las acciones del PIF, esto es, conseguir que la familia se convierta en "compradora" del Programa. A partir de este momento, la intervención va encaminada a conseguir el acercamiento del joven a su familia y viceversa, a través de la generación y desarrollo de nuevas competencias y habilidades propias del ciclo evolutivo familiar en el que se encuentran (familias con hijos adolescentes): habilidades parentales, de comunicación y de negociación.

El proceso de internamiento se inicia con un trabajo dirigido a la reorganización del contexto del menor, donde el ambiente familiar está en el punto de mira. Por lo cual no hay o no debería haber ningún "corte" o aislamiento del núcleo familiar, aunque sí es cierto que comienzan a generarse los reajustes necesarios desde el contacto telefónico que se produce el primer día, y se va trabajando a medida que se celebran las primeras entrevistas y los primeros encuentros.

Desde ese primer momento, tanto el menor como su familia son invitados a participar en el PIF, donde se interviene desde un modelo sistémico, y que consta de las siguientes fases:

- Obtención de información del núcleo familiar: como paso previo, se recaba información relativa tanto de la estructura como de la dinámica familiar, estilos educativos, límites y normas, relaciones de poder, formas de comunicación y otros hitos propios de los sistemas familiares, con el fin de diseñar la posterior actuación orientativa. Esto se hace a través de las instituciones y de los profesionales que han estado implicados en el caso (equipo técnico y educativo de fiscalía, escuela, protección de menores, servicios sociales comunitarios, educadores de medio abierto...) y se completa en momentos posteriores con las entrevistas a las familias.

- Entrevista inicial del equipo técnico y educativo con la familia o tutor del menor, y/o personas vinculadas: se realiza una primera entrevista para contrastar la información obtenida por los agentes mencionados, se solicita información adicional de utilidad para planificar el trabajo con el adolescente, se inicia la relación terapéutica, y se programa una forma de trabajo conjunto con objetivos a corto y medio plazo para la familia y para el menor. Se acuerdan las sesiones posteriores.

- Visita al domicilio familiar del menor: el personal del equipo técnico se desplaza al domicilio para valorar las condiciones del entorno físico donde se incorporará el menor, procurando construir la relación empática con el acercamiento a la familia fuera de la institución.

- Reuniones periódicas con el equipo técnico: durante las cuales se realiza la intervención y se va evaluando el grado de cumplimiento de los objetivos. Se programan mensualmente con una duración aproximada de una hora, en las que se abordan las necesidades que van surgiendo, y valoran los reajustes necesarios.

- Reunión final: se valora exhaustivamente el proceso que ha seguido el menor y se determina su inserción en el entorno familiar y social.

- Seguimiento: es responsabilidad de otra institución externa al Centro. Recae en manos de los delegados de libertad vigilada, educadores sociales que forman parte de la Dirección General de Menores y Familia del Gobierno de las Islas Baleares.

Completando estas fases del Programa, y debido a las características de la situación (privación de libertad), se ofrecen:

- Llamadas telefónicas: se permite al menor mantener un contacto familiar a través de llamadas telefónicas, dos semanales desde el Centro bajo la supervisión del educador. Por su parte, la familia podrá realizar también llamadas dentro de un horario estipulado y pactado.

- Visitas: el Centro dispone también de un horario de visitas de los familiares que son supervisadas por un profesional del mismo.

- Salidas familiares: se planifican salidas familiares en función del horario laboral y la disponibilidad de los familiares. Antes de cada salida se pactan una serie de compromisos que han de asumir tanto las familias como los menores, quedando reflejado por escrito y firmado por los implicados (por ejemplo, la realización de deberes escolares del aula, o no discutir durante las salidas).

- Taller de familias: se realizan talleres complementarios de competencia emocional y social para los menores y sus familias. A través de sesiones grupales con todos los miembros que acudan, se fomenta su participación activa en el proceso educativo de los jóvenes. El objetivo de estos talleres tiene su razón de ser en la necesidad de incidir en el contexto familiar de los chicos y chicas, fundamental 
para que el retorno familiar cobre eficacia y los cambios que se realicen dentro del sistema familiar perduren en el tiempo al observar beneficios. A través del "reencuadre" se intenta transmitir que el internamiento de un alumno menor o joven supone un acontecimiento traumático para toda la familia, pero que este hecho puede vivenciarse como una oportunidad para que ambas partes adquieran nuevas formas de relación y de convivencia más funcionales. El taller de familias se realiza de manera mensual y finaliza con un espacio de encuentro de familiares y jóvenes. Este espacio es un momento muy enriquecedor ya que los padres (tutores o familiares) pueden compartir, en un ambiente informal de no juicio y no moralización, tiempo con sus hijos, con los compañeros de sus hijos y con otras familias que se encuentran en la misma situación. Con frecuencia se invita a profesionales que imparten módulos y charlas de distintos temas relacionados con la familia, la adolescencia, la educación para la salud (drogas, alimentación...), etc.

- Jornadas de presentación de proyectos y actividades: también se organizan anualmente encuentros escolares para que las familias puedan conocer el trabajo que se hace en el aula escolar. Dichas jornadas se preparan de manera conjunta desde el Programa de Intervención Familiar y el Programa Escolar del Centro.

- Fiestas familiares: al final de cada trimestre se organiza una fiesta a la que se invita a los familiares, donde los propios jóvenes preparan la comida que ofrecen a sus padres, tíos, hermanos... Durante estas fiestas se proyectan vídeos para compartir en familia, se exponen fotografías pinturas, etc., realizadas por ellos durante las actividades en el Centro, y se les entrega a las familias un ejemplar de la revista que también es elaborada por los alumnos.

\section{Medios y recursos}

Como se ha adelantado, la primera fase se desarrolla en las dependencias del Centro, comenzando con llamadas telefónicas y posteriormente con visitas que tienen lugar en una sala habilitada para recibir a las familias (con o sin los menores o jóvenes) e iniciar un proceso de colaboración. Para que se celebre la primera visita se ha de establecer una primera entrevista entre familia y los profesionales del Centro vinculados al PIF y al Programa Escolar sin la presencia del alumno.

En fases posteriores se inicia el trabajo en el contexto de referencia de cada alumno: casa/barrio/zona del núcleo familiar directo y familia extensa. A partir de visitas guiadas, salidas de fin de semana supervisadas o estancias con pernocta sin supervisión, pero bajo contrato familiar, se va trabajando con el usuario y con las familias cómo tienen que ser esas salidas y qué tipo de relaciones y situaciones se tienen que ir generando.

Además, todo este proceso se ve enriquecido por otros profesionales (técnicos de los Servicios Sociales o del Departamento de Protección de Menores) que aportan pautas, apoyo y asesoramiento en el proceso de reinserción. La mayoría de los menores internos ya han tenido contacto con servicios/agentes de esta red social. Igualmente se extiende una invitación a profesorado universitario (hasta el momento de las Universidades de las Islas Baleares, de Burgos y de Vic, por el perfil académico de los profesionales convocados) para trabajar de forma conjunta en materia de investigación inclusiva, capacitación e innovación (Álvarez-Arregui, Rodríguez-Martín, Agudo, y Arreguit, 2017) en el ámbito de los menores y jóvenes que han cometido delitos.

\section{Evaluación}

Durante el desarrollo de los talleres, jornadas y fiestas, el equipo técnico y educativo registra en un cuaderno de campo las valoraciones espontáneas realizadas por los miembros asistentes. Al finalizar la medida de internamiento, las familias de los jóvenes responden a un cuestionario confidencial y anónimo de satisfacción administrado por la Fundación S’Estel. Tanto es así que carece de preguntas sociodemográficas. El cuestionario consta de ocho preguntas, teniendo algunas de ellas diferentes apartados, y una casilla de comentarios abiertos. En relación al trabajo familiar, se recogen dos preguntas: una se dirige a conocer el grado de participación y satisfacción con el programa de familias; y la otra busca posibles vías de intervención para la mejora de la relación familiar desde el punto de vista de las propias familias y la repercusión del proceso general de internamiento en la familia.

El cómputo de menores internados en los cuatro últimos años se recoge en la Tabla 1 (los datos referidos a 2017 son parciales). En todos los casos los menores tienen familias reconocidas o legales. Se considera participación en el PIF, tal y como se ha indicado en epígrafes anteriores, que las familias hayan acudido a las entrevistas de intervención o reuniones periódicas con el equipo técnico-educativo, y además, hayan participado en al menos un taller complementario por trimestre.

Tabla 1. Perfil de los participantes.

\begin{tabular}{lcccc}
\hline & \multicolumn{4}{c}{ Años } \\
& 2014 & 2015 & 2016 & 2017 \\
\hline Menores internos & 26 & 24 & 35 & $(27)$ \\
Familias legales reconocidas & 26 & 24 & 35 & $(27)$ \\
$\begin{array}{l}\text { Familias que han participado en el PIF } \\
\text { Cuestionarios cumplimentados: }\end{array}$ & 11 & 8 & 15 & $(12)$ \\
Familias participantes en el PIF & 11 & 8 & 15 & $(9)$ \\
Familias no participantes en el PIF & 3 & & & $(3)$ \\
\hline
\end{tabular}

Por otro lado, de los cuestionarios de satisfacción cumplimentados por las familias participantes en el Programa se extraen los datos mostrados en las Tablas 2 y 3 :

Tabla 2. Valoraciones recibidas del PIF.

\begin{tabular}{lcccc}
\hline & \multicolumn{4}{c}{ Años } \\
Valoración & 2014 & 2015 & 2016 & 2017 \\
\hline 5 & 5 & 6 & 15 & $(4)$ \\
4 & 4 & 2 & & $(5)$ \\
3 & 2 & & & \\
2 & & & & \\
1 & & & & \\
0 & 11 & 8 & 15 & $(9)$ \\
Total de valoraciones & 4,27 & 4,75 & 5,00 & $(4,44)$ \\
Valoración media & & & & \\
\hline
\end{tabular}

Tabla 3. ¿Consideras que el internamiento de tu hijo/a menor ha mejorado las relaciones familiares?

(familias participantes en el PIF)

\begin{tabular}{lcccc}
\hline & \multicolumn{4}{c}{ Años } \\
& 2014 & 2015 & 2016 & 2017 \\
\hline $\begin{array}{l}\text { Sí ha mejorado } \\
\text { No ha mejorado }\end{array}$ & 11 & 8 & 14 & (9) \\
No sabe / no contesta & & & 1 & \\
\hline
\end{tabular}


En el momento de elaborar este cómputo aún no había finalizado el año 2017, y de los 27 menores internos, 12 ya habían finalizado sus medidas cautelares o de privación de libertad, culminando el proceso de reinserción, y regresando a su contexto social, familiar y escolar de referencia. De los 15 menores que continuaban internados, había familias participando en el PIF, por lo que aún no se les había facilitado el cuestionario. Las familias de estos 12 menores reinsertados habían cumplimentado el cuestionario. Las nueve que participaron en el PIF consideraron que el internamiento había mejorado las relaciones familiares. Por el contrario, de las tres familias que no participaron en el Programa, una señaló que no habían mejorado las relaciones y las otras dos no contestaron.

De las aportaciones más cualitativas señaladas por las familias en los cuestionarios, los padres reconocen el acompañamiento que el equipo técnico y educativo durante el desarrollo de los Programas:

- "Me he sentido muy acompañado por parte de los maestros y trabajadora social en el proceso formativo de mi hijo" (junio, 2017).

- "Participar del taller de familias me ha ayudado a entender más a mi hija y a no sentirme tan sola" (mayo, 2017).

En las valoraciones de las familias procedentes del cuaderno de campo del equipo técnico y educativo durante la realización de los talleres, se refleja la evolución de los menores en torno al reconocimiento de su rol como alumnado competente:

- "Hacía muchos años que no me sentía orgulloso de mi hijo" (padre tras presenciar cómo presentaba un proyecto de investigación de Ciencias Sociales, marzo, 2016).

- "Nunca me habían hablado de mi hijo como un buen alumno" (mayo, 2015).

- "Me ha sorprendido la profesionalidad de mi niña, se ha tomado súper en serio la presentación del catering" (madre tras una merienda preparada por los alumnos del FPB de cocina, curso del cual es alumna su hija, octubre, 2014).

- "Ahora me ayuda en casa a hacer comidas y cenas, se ha convertido en mi pinche" (madre de un alumno del FPB de cocina durante uno de los talleres, mayo, 2014).

\section{Conclusiones}

La familia cumple una función muy importante dentro del proceso de socialización de los adolescentes (Sanz et al., 2004). Precisamente por esta razón el menor internado necesita la seguridad y sobretodo la afectividad de una familia durante este proceso. Aun así, tal y como hemos podido comprobar, las familias de menores infractores usuarias de los programas de intervención no siempre promueven el establecimiento de vínculos que contribuyen al desarrollo adecuado de habilidades para afrontar la vida cotidiana en la sociedad. Al contrario, en múltiples ocasiones construyen dinámicas generadoras de conflicto que repercuten negativamente en las/los jóvenes que son internados, siendo esto un factor de riesgo que puede generar más desestabilidad, conflictividad y problemas psicosociales (Amarís et al., 2005) e incluso generar depresión como apuntan $\underline{\text { Ibabe et }}$ al. (2014). Este hecho reafirma la importancia y la necesidad de programas de trabajo con las familias como el presentado con la finalidad de que se conviertan en un elemento favorable en el proceso de inclusión.

Los problemas de la delincuencia juvenil deben ser vistos y tratados desde las principales unidades de socialización del jo- ven: la familia, la escuela, los iguales o amigos, y la comunidad (Egido, 2015; Garreta, 2017; López, 2014). Así pues, es importante que los profesionales de las distintas instituciones responsables de llevar a cabo el proceso de reinserción de los menores infractores tengan en cuenta a la familia en sus actuaciones. Los jóvenes infractores no pueden ser tratados de forma aislada si se quieren tener ciertas garantías de éxito en dicho proceso, sino dentro de un marco comunitario más que individual, resaltando en particular una intervención que respete la importancia de los lazos familiares y comunitarios como elemento clave en la reinserción (Funes, 1992; Trotter et al., 2015; Vázquez, 2012).

Tal y como hemos visto en este trabajo, algunas de las prácticas llevadas a cabo para dar apoyo a las familias, y las posibilidades que éstas tienen de intervenir en la educación de sus hijos e hijas, pueden ser limitadas. Es por ello que Collet y Tort (2008) exponen la necesidad de que la participación de las familias esté contemplada y promovida también de forma más concreta y exhaustiva a nivel legislativo. Así como también sería interesante disponer de normativas que dieran apoyo a programas como el presentado para optimizar las relaciones socio-familiares y contribuir al retorno de estos menores, y convertirlos en alumnos competentes y ciudadanos responsables.

Las limitaciones en el Programa descrito se encuentren principalmente en la fase de seguimiento. Por una parte, el cuestionario de satisfacción no contiene preguntas relativas a datos sociodemográficos, lo que dificulta establecer comparaciones a la luz de la literatura científica (por ejemplo, la mayor participación de la madre en el PIF, dato que es referido por el personal del Centro, corroboraría el perfil de autoridad centrada en la figura materna). Por otra parte, la dificultad para conocer información relativa a la reinserción escolar del menor o del propio proceso de reinserción impide una evaluación profunda de la eficacia del mismo, dado que no existe una base de datos que permita el cruce de datos del internamiento y del proceso posterior al mismo (por ejemplo, finalización de estudios). La LORPM tampoco facilita este análisis, al establecer que un menor no puede ser vinculado a ningún centro de menores después de haber cumplido la medida prescrita.

Si bien se están intensificando las coordinaciones pre- y post-internamiento entre los profesionales implicados en el circuito de justicia juvenil, parece imprescindible tener en consideración la idea de elaborar además un protocolo oficial de intercambio de información que permita a equipos técnicos y educativos de las entidades implicadas en la atención a menores, que supervisan las medidas de libertad vigilada, dar continuidad a las intervenciones del menor y su proceso de reinserción.

Finalmente cabe señalar que el hecho de que las familias vean a sus hijos como alumnos con éxito en el sistema educativo y futuros ciudadanos comprometidos hace que la confianza en ellos y en sí mismos aumente. En consecuencia, el proceso formativo debe ir estrechamente ligado al familiar de manera constante, tomando importancia la promoción de unas relaciones eficaces y recíprocas entre familias y profesionales de la escuela (Collet, Besalú, Feu, y Tort, 2014). A esta acción educativa compartida hay que sumarle la participación activa de los agentes sociales comprometidos con la atención a estos menores, para seguir caminando con paso más firme y coordinado hacia una educación inclusiva de éxito.

\section{Referencias bibliográficas}

Ainscow, M., Dyson, A., Goldrick, S., y West, M. (2012). Making schools effective for all: Rethinking the task. School Leadership $\mathcal{E}$ Management, 32(3), 197-213. 
Álvarez-Arregui, E., Rodríguez-Martín, A., Agudo, S., y Arreguit, X. (2017). Inclusive ecosystem model for the management of knowledge, training and innovation: progress, difficulties and challenges. Aula Abierta, 46, 97-104.

Amarís, M., Amar, J., y Jiménez, M. (2005). Dinámica de las familias de menores con problemas psicosociales: el caso del menor infractor y la menor explotada sexualmente. Revista Latinoamericana de Ciencias Sociales, Niñez y Juventud, 3(2), 141174.

Barton, L. (2003). Inclusive education and teacher education: A basis for hope or a discourse of delusion. Conferencia magistral presentada en el Institute of Education Centenary Lectures. Recuperado de https://goo.gl/KJS12x

Barton, L. (2009). Estudios sobre discapacidad y la búsqueda de la inclusividad: Observaciones. Revista de Educación, 349, 137152.

Bolívar, A. (2006). Familia y escuela: dos mundos llamados a trabajar en común. Revista de Educación, 339, 119-146.

Cagigal, V. (Coord.) (2009). La orientación familiar en el ámbito escolar. Madrid: Universidad Pontificia de Comillas.

Collet, J., Besalú, X., Feu, J., y Tort, A. (2014). Escuelas, familias y resultados académicos: un nuevo modelo de análisis de las relaciones entre docentes y progenitores para el éxito de todo el alumnado. Profesorado: Revista de Currículum y Formación del Profesorado, 18(2), 7-33.

Collet, J., y Tort, A. (2008). Espacios de participación. Cuadernos de Pedagogía, 378, 57-60.

Constitución Española (1978). Boletín Oficial del Estado, 311. Referencia: BOE-A-1978-31229.

Coy, E., y Torrente, G. (1997). Intervención con menores infractores: su evolución en España. Anales de Psicología, 13(1), 39-49.

Decreto por el que se establece el currículo de la Educación Secundaria Obligatoria en las Islas Baleares (Decreto 34/2015, de 15 de mayo). Butlletí Oficial de les Illes Balears/Boletín Oficial de las Islas Baleares, núm. 73, 2015, 16 de mayo. Referencia: 8751.

Egido, G.I. (2015). Las relaciones entre familia y escuela. Una visión general. Participación Educativa. Revista del Consejo General del Estado. Segunda Época, 4(7), 11-17.

Fonagy, P., Butler, S., Goodyer, I., Cottrell, D., Scott, S., Pilling, S., Eisler I, Fuggle P, Kraam A, Byford S, Wason J, Haley R., y Haley, R. (2013). Evaluation of multisystemic therapy pilot services in the Systemic Therapy for At Risk Teens (START) trial: study protocol for a randomised controlled trial. Trials, 14(265), 1-19.

Funes, J. (1992). Criterios para la incorporación social de los menores infractores. Encuentros sobre servicios sociales y reinserción social de delincuentes y jóvenes inadaptados. Sevilla: Instituto Andaluz de Servicios Sociales.

Garreta, J. (Coord.) (2017). Familias y escuelas: Discursos y prácticas sobre la participación en la escuela. Madrid: Pirámide.

Germán, I. y Ocáriz, E. (2009). Menores infractores/menores víctimas: hacia la ruptura del círculo victimal. Eguzkilore, 23, 287-300.

Ibabe, I., y Jaureguizar, J. (2011). Perfil psicológico de los menores denunciados por violencia filio-parental. Revista Española de Investigación Criminológica, 9(6), 1-19.
Ibabe, I., Arnoso, A., y Elgorriaga, E., (2014). Behavioral problems and depressive symptomatology as predictors of childto-parent violence. European Journal of Psychology Applied to Legal Context, 6(2), 53-61. doi:10.1016/j.ejpal.2014.06.004.

Lema, C. (2010). El impacto en la educación de la Convención de la ONU sobre los Derechos de las Personas con Discapacidad. En M.A. Casanova y M.A. Cabra (Coords.), Educación y Personas con discapacidad: Presente y Futuro (p. 31-65). Madrid: Fundación ONCE.

Ley Orgánica reguladora del derecho a la educación (LODE) (Ley Orgánica 8/1985, de 3 de julio), Boletín Oficial del Estado, núm. 159, 1985, 4 de julio. Referencia: BOE-A-1985-12978.

Ley Orgánica reguladora de la responsabilidad penal de los menores (LORPM) (Ley Orgánica 5/2000, 12 de enero). Boletín Oficial del Estado, núm. 13, 2000, 2 de enero. Referencia: BOE-A-2000-641.

Ley Orgánica para la mejora de la calidad educativa (LOMCE) (Ley Orgánica 8/2013, 9 de diciembre). Boletín Oficial del Estado, núm. 295, 2013, 10 de diciembre. Referencia: BOE-A-2013-12886.

López, N. (2006). El procés d'inserció socioescolar o socioformatiu per a joves amb mesures judicials de privació de llibertat. En J.R. Lago, G. Riera, O. Pedregosa, y J. Soldevila (Coords.), Cap a una Educación Inclusiva (p. 497-511). Vic: Eumo.

López, R.F. (2014). La participación de las familias en la educación escolar. Madrid: Ministerio de Educación, Cultura y Deporte.

López, N., y Muñoz, M.J. (2007). El trabajo constructivo para la mejora de las relaciones socio-familiares con menores infractores: Centro Socioeducativo de menores "Es Fusteret". En M. Fernández e I. García (Dir.), Orientación Familiar: Violencia Familiar (p. 131-138). Burgos: Universidad de Burgos.

Marchesi, A. (2004). Qué será de nosotros, los malos alumnos. Madrid: Alianza.

Orregoa, J.M., Painob, M., y Fonseca-Pedreroc, E. (2016). Programa educativo «Trampolín» para adolescentes con problemas graves del comportamiento: perfil de sus participantes $\mathrm{y}$ efecto de la intervención. Aula Abierta, 44, 38-45.

Rey, C. (2014). Menores infractores en centros de reforma: intervención y tratamiento psicológico. Derecho y Cambio Social, 37, 1-9.

Sanz, M., Martínez-Pampliega, A., Iraurgi, J., Muñoz-Eguileta, A., Galíndez, G., Cosgaya, L., y Nolte, M. (2004). El conflicto parental y el consumo de drogas en los hijos y las hijas. Bilbao: AEFFA-Dirección de Drogodependencias del Gobierno Vasco.

Stainback, S., Stainback, W., y Moravec, J. (1999). Un currículo para crear aulas inclusivas. En S. Stainback, y W. Stainback (Coords.). Aulas Inclusivas (p. 83-102). Madrid: Narcea.

Trotter, C., Baidawi, S., y Evans, P. (2015). Good Practice in Community-based Supervision of Aboriginal Youth Offenders. Australian Social Work, 68(1), 5-18.

Vásquez, C. (2003). Predicción y prevención de la delincuencia juvenil según las teorías del desarrollo social (social development theories). Revista de Derecho (Valdivia), 14, 135-158.

Vázquez, L.D. (2012). Acercamiento estadístico a la realidad de los menores infractores en México: legislación y crimen organizado, nuevos desafíos. Universitas Psychologica, 11(4), 11051114 . 